\title{
Clinical application of magnetic resonance spectroscopy of the liver in patients receiving long-term parenteral nutrition
}

\author{
J. M. Woodward ${ }^{1}$, A. N. Priest ${ }^{2}$, K. G. Hollingsworth ${ }^{3}$, S. J. Cottee ${ }^{1}$ and D. J. Lomas ${ }^{4}$ \\ ${ }^{1}$ Department of Gastroenterology (Nutrition team), Addenbrookes Hospital, Cambridge, UK, ${ }^{2}$ Departments of Radiology \\ and Medical Physics, Addenbrookes Hospital, Cambridge, UK, ${ }^{3}$ Department of Radiology, University of Cambridge, UK \\ and ${ }^{4}$ Department of Radiology, Addenbrookes Hospital, and University of Cambridge, Cambridge, UK
}

Liver disease remains a significant complication of intestinal failure treated with parenteral nutrition. Progression to cirrhosis and portal hypertension can be rapid and is a major indication for intestinal transplantation in both children and adults. Detection may be delayed as: (1) asymptomatic liver enzyme abnormalities are commonly associated with parenteral nutrition; (2) the anatomy of patients with intestinal failure may preclude the more typical presentations of portal hypertension and (3) parenteral nutrition support may mask the nutritional consequences of cirrhosis. The aetiology is likely to be multifactorial; choline deficiency has been implicated in adults and benefits have been demonstrated from supplementation. This pilot study was initiated to determine the value of a non-invasive test in detecting liver complications in patients receiving long-term parenteral nutrition.

Magnetic resonance spectra were obtained from six female patients under the care of the Addenbrooke's Hospital Nutrition Team (ages 50 (SD 13) years, BMI 20.5 (SD 3.1) kg/m², length of TPN 9 (SD 4) years) and from six healthy female volunteers (age 35 (SD 13) years, BMI $23(\mathrm{SD} 2.91) \mathrm{kg} / \mathrm{m}^{2}$ ). The study was approved by the local research ethics committee. All patients were receiving less than $1 \mathrm{~g}$ lipid/ $\mathrm{kg}$ perd. The scans were acquired after a fast of at least $6 \mathrm{~h}$ using a 1.5 Tesla scanner (Signa Excite III, GE Healthcare). Proton $\left({ }^{1} \mathrm{H}\right)$ spectra and phosphorus $\left({ }^{31} \mathrm{P}\right)$ spectra were acquired from the right lobe of the liver. Reconstruction of spectra by data recombination allowed peak areas to be quantified before and after subtraction of water and lipid peaks. $\mathrm{CH}, \mathrm{CH}_{2}$ and $\mathrm{CH}_{3}$ peak areas were summed for total lipid quantification, The choline peak area was calculated following water and lipid peak subtraction and expressed as a ratio choline: water. Phosphomonoester (PME) and phosphodiester (PDE) peak areas were calculated from $\left({ }^{31} \mathrm{P}\right)$ spectra. Results were analysed using Student's $t$ test with a significance level of $P<0.05$. Results are presented in the Table.

\begin{tabular}{|c|c|c|c|c|c|c|}
\hline & \multicolumn{2}{|c|}{ Control } & \multirow{2}{*}{$\begin{array}{l}\text { Control } \\
\text { Range }\end{array}$} & \multicolumn{2}{|c|}{ Patients } & \multirow{2}{*}{$\begin{array}{l}\text { Patients } \\
\text { Range }\end{array}$} \\
\hline & Mean & SD & & Mean & SD & \\
\hline Lipid:water & 0.02 & 0.019 & $0.0048-0.055$ & $0.143 *$ & 0.173 & $0.033-0.478$ \\
\hline Choline $:$ water $\times 1000$ & 1.58 & 0.56 & $0.83-2.34$ & 0.88 & 0.71 & $0.13-2.14$ \\
\hline PME:PDE & 0.205 & 0.042 & $0.169-0.284$ & 0.262 & 0.076 & $0.145-0.343$ \\
\hline
\end{tabular}

* Significance at $P<0.05$.

A significant increase in hepatic lipid deposition is demonstrated in the subjects compared to controls despite close matching for BMI. There was an association with serum alkaline phosphatase levels, but not with transaminase levels. There was no inverse correlation of liver choline levels with fat deposition when based on choline: water ratios rather than choline:lipid ratios. Two patients with PME:PDE ratios $>0.3$ and outside $3 \mathrm{SD}$ of the control mean went on to have liver biopsies that demonstrated cirrhosis.

Magnetic resonance spectroscopy is a non-invasive, non-ionising radiation test that can be used to semi-quantitatively measure hepatic lipid accumulation, to select patients at high risk of advanced disease who should undergo liver biopsy, and will help in the assessment of patients considered for intestinal transplantation. If validated in a larger population, sequential magnetic resonance spectroscopy may play a role in assessing therapeutic intervention to reduce steatosis and may help contribute to our understanding of the aetiology of intestinalfailure-associated liver disease. 